\title{
A High Body Roundness Index Is More Closely Associated With Urinary Albumin-creatinine Ratio Than Traditional Adiposity Indices in Chinese Population: a Cross-sectional Report From the Reaction Study
}

\section{Yun Wang}

Graduate School, Chinese PLA General Hospital, Beijing

Jie Wang

Department of Endocrinology, Beijing Chao-Yang Hospital, Capital Medical University, 8 Gongren

Tiyuchang Nanlu, Chaoyang District, Beijing

Yang Liu

Graduate School, Chinese PLA General Hospital, Beijing

\section{Binqi Li}

School of Medicine, Nankai University, No. 94 Weijin Road, Tianjin

\section{Kang Chen}

Department of Endocrinology, First medical center of PLA General Hospital, Beijing

\section{Weiqing Wang}

Shanghai National Research Centre for Endocrine and Metabolic Diseases, State Key Laboratory of

Medical Genomics, Shanghai Institute for Endocrine and Metabolic Diseases, Ruijin Hospital『Shanghai

\section{Zhengnan Gao}

Dalian Central Hospital, Dalian, Liaoning

\section{Xulei Tang}

First Hospital of Lanzhou University, Lanzhou, Gansu

\section{Li Yan}

Zhongshan University Sun Yat-sen Memorial Hospital, Guangzhou, Guangdong

\section{Qin Wan}

Southwest Medical University Affiliated Hospital, Luzhou, Sichuan

\section{Zuojie Luo}

First Affiliated Hospital of Guangxi Medical University, Nanning, Guangxi

\section{Guijun Qin}

First Affiliated Hospital of Zhengzhou University, Zhengzhou, Henan

\section{Lulu Chen}

Wuhan Union Hospital, Huazhong University of Science and Technology, Wuhan, Hubei 


\section{Yiming Mu ( $\square$ muyiming@301hospital.com.cn )}

Department of Endocrinology, First medical center of PLA General Hospital, Beijing

\section{Research Article}

Keywords: body roundness index 1 , albuminuria2, body fat distribution3, Chinese population4, chronic kidney disease 5

Posted Date: January 28th, 2022

DOI: https://doi.org/10.21203/rs.3.rs-1294691/v1

License: (c) (1) This work is licensed under a Creative Commons Attribution 4.0 International License. Read Full License 


\section{Abstract}

\section{Objective:}

Body roundness index (BRI) has been proposed to be a promising index of body fat distribution. The association between BRI and increased albuminuria is unclear. Therefore, the aim of this study is to examine the discriminative ability of BRI for increased albuminuria and compare the association of BRI and traditional adiposity indices (body mass index: BMI, waist circumstance: WC, waist-to-hip ratio: WHR) with albuminuria.

\section{Methods:}

This cross-sectional study was nested in an ongoing REACTION study. A total of 43591 participants aged over 40 years were recruited across seven different regional provinces. Increased albuminuria was defined as urinary albumin to creatinine ratio (UACR) $\geq 30 \mathrm{mg} / \mathrm{g}$. Multiple logistic regression analyses were performed to detect the association between BRI and UACR and compare the discriminative ability of traditional (BMI, WC, WHR) and new adiposity indices (waist-to-height ratio: WHtR, BRI) with UACR.

\section{Results:}

Participants with increased UACR exhibited increased age, blood pressure, blood glucose, poor control of lipid level, decreased estimated glomerular filtration rate (eGFR)『and higher prevalence of diabetes, hypertension and cardiovascular events. Multiple logistic models showed that compared with traditional adiposity indices (BMI, WC, WHR), the BRI index remained significantly associated with UACR especially in women, indicating the strong discriminative power for increased albuminuria. Stratified analysis revealed that the strong positive association of the BRI index with increased albuminuria also occurred in people who were young, women, poor control of hypertension and blood glucose, and eGFR $\geq 90$ $\mathrm{ml} / \mathrm{min} / 1.73^{2}$.

\section{Conclusions:}

The BRI index were closely associated with increased albuminuria in the Chinese population.

\section{Introduction}

Elevated urinary albumin to creatinine ratio (UACR) has been recommended as an indicator of chronic kidney disease (CKD), ${ }^{1}$ and a key risk factor for many chronic diseases. Romero-Aroca et al. indicated that compared with the estimated glomerular filtration rate (eGFR), UACR shows a stronger association with diabetic retinopathy. ${ }^{2}$ Moreover, UACR can not only be predictive of cardiovascular disease (CVD), but also has important value in evaluating the left ventricular dysfunction. ${ }^{3} \mathrm{~A}$ recent study has demonstrated that UACR is closely associated with preeclampsia and could be used as an early predictor of preeclampsia in the pre-gestational diabetic population. ${ }^{4}$ Furthermore, UACR has been confirmed 
associated with vascular damage. Not only in diabetic population, but also in patients with acute lacunar infarction, increased UACR is a significant indicator for cerebral small vessel diseases. ${ }^{5}$

A growing body of evidence have reported that obesity is a key driver of CKD, 6,7 and increased UACR as an early indicator of CKD could effectively reflect the injury of glomerular and tubular cell function. Recently, body mass index (BMI), an easily applicable measure of obesity, has been reported to be closely associated with CKD progression in hypertensive patients. ${ }^{8}$ Framingham study cohort has suggested that high BMI could be independently predicting of the renal dysfunction, ${ }^{9}$ and it is valuable in predicting the onset of albuminuria. ${ }^{10}$ However, several studies has demonstrated that it is the accumulation of visceral fat rather than subcutaneous fat that promotes the development of CKD. ${ }^{11-13}$ Although BMI, waist circumstance (WC) and waist-to-hip ratio (WHR) are commonly used adiposity indices with the advantages of convenience, they provided limited information on the body fat distribution and body shape. ${ }^{14-16}$ Thus, it is important to find a simple and effective screening tool for albuminuria to reduce the incidence of CKD.

In recent years, a new anthropometric index, body roundness index (BRI), have been proposed as an indicator of body fat distribution and body shape, because it has a strong and stable relationship with the abdominal adipose accumulation. BRI, a predictor of body fat and visceral adipose tissue volume, has been confirmed to have the potential to identify the status of diabetes. ${ }^{17-19}$ It is well known that diabetes is an additional cause of CKD and obesity is the main pathogenetic pathway linking diabetes and CKD. However, the relationship between fat distribution and albuminuria is still unknown.

To the best of our knowledge, there are limited studies involving the relationship between BRI and albuminuria and it is still unclear that whether traditional adiposity indices or newly developed adiposity indices are more valuable in estimating the renal dysfunction. Therefore, the aim of this study is to examine the association between BRI with UACR and compare the discriminative ability of the traditional and new anthropometric indices for UACR.

\section{Materials And Methods}

\section{Study population and design}

This cross-sectional study was nested in a longitudinal REACTION (Risk Evaluation of Cancers in Chinese diabetic Individuals) study, which was designed to investigate the association between T2DM and the risk of cancer among the Chinese population. Details of the REACTION study have been previously reported. ${ }^{20,21}$ The present study used baseline investigation data from seven centers across China. Initially, a total of 45130 participants aged over 40 years were recruited from May 2011 to December 2011. Participants diagnosed with kidney or other related diseases, those using ACEI/ARB medicines and those with missing data were excluded as shown in Figure 1. Finally, 43591 participants were enrolled in the present study. 
Before carrying out the investigation, the staff received extensive training, including the standardized questionnaire and data collection. The protocol of present study was approved by the Committee on Human Research at Rui-Jin Hospital affiliated with the School of Medicine, Shanghai Jiao Tong University. This study adhered to the principles of the Declaration of Helsinki. Written informed consents were obtained from all participants before data collection.

\section{Data collection}

Data collection were performed by the same trained staff, according to standardized operational procedures. All the participants received the same comprehensive examinations including a standard questionnaire, anthropometric measurements, venous blood collection, and 75-g oral glucose tolerance test (OGTT) or bread meal test. The questionnaire included the history of diabetes, hypertension, acute/chronic nephritis, nephritic syndrome, kidney stones, CVD, diabetes, alcohol habits, and smoking habits. Regular smokers were defined as those who smoked at least one cigarette per day. Occasional smokers were participants who smoked less than one cigarette per day or less than 7 cigarettes per week. Regular drinkers were defined as participants who consumed alcohol at least once a week for over six months. Occasional drinkers were defined as participants who drank less than once a week.

Height, weight, WC and hip circumstance (HC) were measured by the same well-trained staff after participants were required to wear light clothing and take off the shoes. Height and weight were clinically measured in light clothing, using the same device with the precision of $0.01 \mathrm{~m}$ and $0.1 \mathrm{~kg}$, respectively. WC was measured by the same staff placing a tape horizontally between the inferior costal margin and the superior border of iliac crest at the end of expiration and that was measured to the nearest $0.01 \mathrm{~m}$. $\mathrm{HC}$ was recorded at the maximum circumference over the buttocks, to the nearest $0.01 \mathrm{~m}$.

After resting for five minutes, participants' blood pressure and pulse were measured three times with in one-minute intervals by the same staff. The average of blood pressure was calculated and used in the statistical analysis. The pulse rate was measured while the blood pressure was recorded. After 8-10 of fasting overnight, the first fasting blood samples of all the participants were obtained. Patients without a history of T2DM underwent a $75 \mathrm{~g} \mathrm{OGTT}$; they were required to drink $300 \mathrm{~mL}$ of a glucose solution containing $75 \mathrm{~g}$ of glucose within 5 minutes. After 2 hours, the second venous blood sampling was obtained by the same well-trained staff. Fasting blood glucose (FBG), $2 \mathrm{~h}$ post-load blood glucose (PBG), serum triglycerides $(\mathrm{TG})$, total cholesterol $(\mathrm{TC})$, high-density lipoprotein cholesterol (HDL), low-density lipoprotein cholesterol (LDL), and serum creatinine ( $\mathrm{Cr}$ ), Haemoglobin A1c (HbA1c), alanine transferase (ALT), aspartate transferase (AST), and gamma-glutamyl transferase (GGT) were respectively measured in every center.

The variables were defined as follows: hypertension (any self-reported history of hypertension or systolic blood pressure SBP $\geq 140 \mathrm{mmHg}$ or diastolic blood pressure DBP $\geq 90 \mathrm{mmHg}$ ), T2DM (FBG $\geq 7.0$ $\mathrm{mmol} / \mathrm{L}$ or $\mathrm{PBG} \geq 11.1 \mathrm{mmol} / \mathrm{L}$ simultaneously or any self-reported history of diabetes), cardiovascular events (any self-reported history of coronary heart disease, stroke, and myocardial infarction). According to the WHO criteria, prediabetes was defined as follows: $6.1 \leq \mathrm{FBG}<7.0 \mathrm{mmol} / \mathrm{L}$ or $2 \mathrm{~h} \mathrm{PBG}<11.1$ 
$\mathrm{mmol} / \mathrm{L}$. Based on WHO criteria, prediabetes was further divided into 3 groups as follows: impaired fasting glucose (IFG): $6.1 \leq \mathrm{FBG}<7.0 \mathrm{mmol} / \mathrm{L}$ and $\mathrm{PBG}<7.8 \mathrm{mmol} / \mathrm{L}$; impaired glucose tolerance (IGT): $\mathrm{FBG}<6.1 \mathrm{mmol} / \mathrm{L}$ and $7.8 \leq \mathrm{PBG}<11.1 \mathrm{mmol} / \mathrm{L}$; and $\mathrm{IFG}+\mathrm{IGT}: 61 \leq \mathrm{FBG}<7.0 \mathrm{mmol} / \mathrm{L}$ and $7.8 \leq \mathrm{PBG}$ $<11.1 \mathrm{mmol} / \mathrm{L}$. eGFR was calculated based on the Chronic Kidney Disease Epidemiology Collaboration (CKD-EPI). ${ }^{22}$

\section{Definition of UACR group and anthropometric indices}

The concentration of urine albumin and creatinine were measured by collecting the first urine specimens in the morning. The definition of UACR was calculated using the following formula: urinary albumin $(\mathrm{mg})$ /urinary creatinine $(\mathrm{g})$. All seven centers used the same normal value range and unit of measurement as the following, normo-albuminuria: $<30 \mathrm{mg} / \mathrm{g}$; increased albuminuria $\geq 30 \mathrm{mg} / \mathrm{g}$.

$\mathrm{BMI}$ was calculated using the formula: $\mathrm{BMI}=$ weight $\mathrm{kg} /$ height $\mathrm{m}^{2}$. WHtR was calculated as the WC divided by the height. $A B S I$ and $B R I$ were calculated using the following formula: $B R I=364.2-365.5 \times(1-$ $[\mathrm{WC} / 2 \pi]^{2} /[0.5 \times$ height2) $1 / 2$. The BRI score was converted to a $z$-score using the following formula: BRI

minus $\mathrm{BRI}$ mean divided by BRI SD. BMI, WC, WHR, WHtR were also transformed to z-score by the same equation. Given difference of the unit change in the logistic regression analysis, the z-score were conducted to made the comparison meaningful. ${ }^{23}$

\section{Statistical analysis}

The statistical analyses were performed using Empower(R) (www.empowerstats.com, X\&Y Solutions Inc., Boston, MA) and R (http://www.Rproject.org). A p value $<0.05$ (2-sided) was considered statistically significant.

Data were presented as median (25th percentile-75th percentile) for continuous variables of non-normal distribution. Category variables were presented as percentage (\%). Differences in the continuous variables among the two subgroups of UACR were analyzed using the Kruskal-Wallis test. The category variables were tested using the chi-square test. Multiple logistic regression analysis was conducted to detect the association of traditional and new adiposity indices with UACR. The conventional risk variables related to renal dysfunction were adjusted, ${ }^{24,25}$ and confounding factors that when added to the model, changed the matched odds ratio (OR) by at least $10 \%$ were also selected for adjustment. Model I was adjusted for age and sex. To further correct the effects of the confounding factors, model II was adjusted for centers, age, sex, BMI, SBP, DBP, FBG, PBG, HbA1c, HDL, LDL, TG, GGT, eGFR, pulse, centers, smoking status, drinking status, and medication history. In order to thoroughly investigate the association and compare the difference in different levels of age, sex, eGFR, the control of blood pressure and glucose, stratified analyses were performed based on the subgroups of age (Age $<55$ years, 55-64 years, $\geq 65$ years), sex (men, women), the subgroup of eGFR (eGFR $\geq 90 \mathrm{~mL} / \mathrm{min}$ per $1.73 \mathrm{~m}^{2}$, eGFR $<90 \mathrm{~mL} / \mathrm{min}$ per $1.73 \mathrm{~m} 2$ ), blood glucose (normal: FBG $<7.0 \mathrm{mmol} / \mathrm{L}$ and/or PBG $<11.1 \mathrm{mmol} / \mathrm{L}$, prediabetes : IFG: $6.1 \leq \mathrm{FBG}<7.0$ $\mathrm{mmol} / \mathrm{L}$ and $\mathrm{PBG}<7.8 \mathrm{mmol} / \mathrm{L}, \mathrm{IGT}: \mathrm{FBG}<6.1 \mathrm{mmol} / \mathrm{L}$ and $7.8 \leq \mathrm{PBG}<11.1 \mathrm{mmol} / \mathrm{L}$, IFG + IGT: $6.1 \leq$ 
FBG $<7.0 \mathrm{mmol} / \mathrm{L}$ and $7.8 \leq \mathrm{PBG}<11.1 \mathrm{mmol} / \mathrm{L}$,diabetes: $\mathrm{FBG} \geq 7.0 \mathrm{mmol} / \mathrm{L}$ and $\mathrm{PBG} \geq 11.1 \mathrm{mmol} / \mathrm{L}$ or any self-reported history of diabetes), blood pressure (normal blood pressure $\triangle \mathrm{SBP}<140 \mathrm{mmHg}$ and diastolic blood pressure $\mathrm{DBP}<90 \mathrm{mmHg}$, hypertension $\triangle \mathrm{SBP} \geq 140 \mathrm{mmHg}$ or DBP $\geq 90 \mathrm{mmHg}$ or any self-reported history of hypertension).

\section{Results}

\section{Demographic Data and Hematologic Parameters of the study population}

A total of 43591 participants with a median age (Q1-Q3) of 57.51 (52.19-63.90) years were included in this study, including 13119 (30.10\%) men and 30472 (69.90\%) women as shown in Table 1. Table 1 shows the basic characteristics based on UACR range (UACR $<30$ or $\geq 30 \mathrm{mg} / \mathrm{g}$ ). Participants with increased UACR had increased age, SBP, DBP, pulse, FBG, PBG, HbA1c, AST, GGT, TG, eGFR, BMI Z score, WC Z score, WHtR Z score, WHR Z score, BRI Z score, as well as decreased TG, LDL, HDL, eGFR. Participants with the increased age have the higher risk of increased UACR. The prevalence of T2DM, hypertension, and CVD events were respectively higher in participants with increased UACR (T2DM: 28.05 $\%$ VS $13.11 \%$, Hypertension: $60.04 \%$ VS $28.05 \%$, CVD events: $8.82 \%$ VS 4.88\%, P<0.001).

\section{Associations between adiposity indices and the risk of increased UACR in total population}

Table 2 shows the significant association between all adiposity indices and UACR in the total population in Model I. After further adjusting for confounding factors in model II, the association between newly developed adiposity indices (WHtR and BRI) remained significant, indicating the stability of the association between WHtR, BRI and increased UACR (WHtR: OR: 1.08, 95\% Cl 1.02-1.13 P=0.0075; BRI: OR: $1.22,95 \% \mathrm{Cl} 1.11-1.33 \mathrm{P}=0.0001)$. However, the significant association between $\mathrm{BMI}, \mathrm{WHR}, \mathrm{WC}$ and increased UACR vanished, suggesting the inferior discriminative ability for albuminuria.

\section{Associations between adiposity indices and increased UACR in stratified analyses}

To verify the discriminative power of all adiposity indices for albuminuria, stratified analyses were performed in subgroups as shown in Table 3. In younger participants (age $<65$ years), BRI was positively associated with the increased UACR, whereas other indices did not present a significant association with increased UACR in Model II ( BRI: age < 55 years: OR: 1.41, 95\% Cl 1.20-1.66 $\mathrm{P}=0.0001 ; 55 \geq$ age <64years: OR: $1.41,95 \% \mathrm{Cl} 1.03-1.38 \mathrm{P}=0.0203$ ). In women, only WHtR and BRI showed a significant association with increased UACR and the discriminative ability of BRI for UACR was superior to WHtR (BRI: OR: 1.29, 95\% Cl 1.16-1.43 P<0.0001VS WHtR: OR: 1.08, 95\% Cl 1.02-1.15 P=0.0083). However, no significant association between BRI and UACR was found in men. Of note is the gender effect of BRI on UACR risk. In stratified analysis, a significant association between BRI and UACR was also observed in both hypertension and diabetes subgroups, but the association was more superior in those without hypertension and those with diabetes (Hypertension Yes: OR:1.14 95\% Cl 1.01-1.28 P=0.0341VS Hypertension No: OR:1.29 95\% Cl 1.12-1.48 P=0.0004; Diabetes Yes: OR:1.33 95\% Cl 1.13-1.57 P=0.0007 VS Diabetes No: OR:1.17 95\% Cl 1.07-1.28 P=0.0009), indicating the additional risk of blood glucose on 
increased albuminuria. To further explore the association of the effect of blood glucose on UACR, the participants with prediabetes were divided into IFG, IGT, and IFG+IGT. Table 4 shows that only BRI had a significant association with increased UACR in the IGT and IGT+IFG groups (IGT: FBG $<6.1 \mathrm{mmol} / \mathrm{L}$ and $7.8 \leq \mathrm{PBG}<11.1 \mathrm{mmol} / \mathrm{L}:$ OR: $1.20,95 \% \mathrm{Cl} 1.08-1.33, \mathrm{P}=0.0005$; IGT+IFG: $61 \leq \mathrm{FBG}<7.0 \mathrm{mmol} / \mathrm{L}$ and $\mathrm{i} 7.8 \leq \mathrm{PBG}<11.1 \mathrm{mmol} / \mathrm{L}: \mathrm{OR}: 1.34,95 \% \mathrm{Cl} 1.03-1.74, \mathrm{P}=0.0320$ ), suggesting the additional risk of poor control of $2 \mathrm{~h}$ post-load blood glucose on increased UACR. Moreover, to thoroughly verify the association of BRI with UACR, stratified analysis was also conducted based on different renal functions, and we found similar results at different level of eGFR. When participants had a normal eGFR $\geq 90 \mathrm{~mL} / \mathrm{min}$ per $1.73 \mathrm{~m} 2$, the odds of having an increased UACR positively in participants with increased BRI (BRI: OR:1.26, 95\% Cl 1.13-1.41, $\mathrm{P}<0.0001)$. However, no significant association was found in participant with eGFR $<90 \mathrm{~mL} / \mathrm{min}$ per $1.73 \mathrm{~m} 2$. The above results show that compared with traditional adiposity indices (BMI, WC, WHR), the new adiposity index BRI presented a superior discriminative ability for albuminuria, suggesting the advantage of BRI as an easy and effective screening tool for early kidney failure.

\section{Discussion}

\section{Main findings}

In the present study, we found that BRI was positively associated with UACR and showed a superior discriminative ability for UACR than traditional adiposity indices (BMI, WHR and WC), suggesting its advantage of being an accurate discriminator for the risk of albuminuria to early predict renal lesion. Stratified analysis revealed that participants with higher BRI were more likely to have albuminuria than those with lower BRI, especially in participants who were young (age $<60$ years), those with abnormal blood glucose ( $F B G \geq 7.0 \mathrm{mmol} / \mathrm{L}$ and $\mathrm{PBG} \geq 11.1 \mathrm{mmol} / \mathrm{L}$ ), and those with normal blood pressure (SBP $\geq 140 \mathrm{mmHg}$ or diastolic blood pressure DBP $\geq 90 \mathrm{mmHg}$ ) and eGFR (eGFR $\geq 90 \mathrm{~mL} / \mathrm{min}$ per $1.73 \mathrm{~m} 2$ ). To the best of our knowledge, the is the first multicenter, large sample investigation to explore the association between BRI and UACR in the Chinese elderly population. Therefore, effective prevention and intervention are important for UACR, and modification of the body fat distribution and body shape may contribute to effectively reduce the incidence of adverse events in participants with poor control of blood glucose and blood pressure.

It is well known that obesity is a leading cause of chronic diseases, including CVD, T2DM, hypertension and CKD. Considering the use of computed tomography (CT) and magnetic resonance imaging (MRI) to detect body composition is accurate but expensive and inconvenient, previous studies proposed to use anthropometric indices to investigate the association between obesity and the risk of chronic diseases. Traditionally, BMI is a commonly recommended anthropometric index of the body fat. BMI has been reported to be valuable in predicting increased UACR.9 As well, it is closely associated with CKD among hypertensive patients as a predictor of the onset of albuminuria. Conversely,8,10 other studies opposed that BMI only indicates general obesity with less accuracy but not abdominal body fat, which plays a more significant role in the progression of chronic diseases.26 Emerging evidence supported that it is abdominal obesity rather than general obesity that has superior predictive value for CVD risk.27 Although 
BMI has been the most commonly used adiposity index, it has limited power to distinguish peripheral from central body fat, fat and lean mass.28,29 Therefore, WC and WHR have been proposed as surrogate indices for abdominal obesity. Previous studies found that both WC and WHR are better predictors of CVD risk than BMI.30-32 A recent study found WC to be more strongly associated with cardiometabolic risks than BMI, while another meta-analysis of 32 studies suggested WC, WHR and BMI have similar associations with the incidence of T2DM.33,34 In this present study, no significant difference was observed among WC, WHR, and BMI, which is consistent with previous studies. The results might be partially explained by the following reasons. First, WC and WHR have the insufficient capability of distinguishing visceral fat from subcutaneous fat, resulting in overestimating the visceral fat tissue in subjects. Second, WC and WHR may underestimate or overestimate the visceral fat without the consideration of height, which is an indicator of nutritional status during childhood.

In recent years, WHtR was also found to be a better screening tool for cardiometabolic risk factors than $\mathrm{BMI}$ and WC in a meta-analysis.32 A cross-sectional study revealed that WHtR was closely associated with cardiometabolic risk factors. 35 Moreover, another case-control study based on Chinese population found that WHtR showed the superior value in the assessment of CKD than BMI and WC, which is in line with our findings. 34

$\mathrm{BRI}$, a novel and promising obesity-related index囚combines height and WC and has been used to quantify the body shape and predict visceral fat tissue volume.20 BRI has been proven to be more valuable in indicating body fat and visceral fat tissue than BMI and WC.20 Some studies investigated that BRI is a better predictor of hyperuricemia than BMI in women. It is a superior index to investigate the association between obesity and hyperuricemia than WC and WHtR. Similarly, another cross-sectional study demonstrated that BRI presents a high predictive value to detect dyslipidemia in women.36,37 However, there is limited evidence on the comparison of the discriminative ability of BRI with other adiposity indices in identifying the risk of UACR. This present study was the first to access the superior ability of BRI to identify the risk of increased UACR compared with the traditional adiposity indices, indicating the advantage of BRI as an accurate and stable indicator of albuminuria.

Though the mechanism underlying the relationship between obesity and albuminuria is complex and unclear, it may be partially explained by the following reasons. It is well established that obesity can result in hemodynamic, hormonal and metabolic changes. First, obesity can induce increased renal plasma flow, glomerular pressure and filtration fraction, resulting in an increase in albuminuria.38,39 Second, it is reported that obesity activates the sympathetic nervous system and renin-angiotensin system (RAS) and physical compression of the kidneys, resulting in the increase of renal tubular sodium reabsorption and volume expansion, especially in the presence of visceral obesity. In addition, obesity can cause renal vasodilation and glomerular hyperfiltration. The results of these changes further lead to glomerular injury and urinary protein excretion.40 Third, the relationship between visceral obesity and albuminuria may be linked to insulin resistance (IR). Accumulating evidence suggested that a characteristic feature of visceral obesity is increased free fatty acid (FFA) levels, which may contribute to the peripheral IR and dyslipidemia.41 The podocyte insulin signaling plays an important role of the 
maintenance of the integrity of the glomerular filtration barrier. IR and lipo-toxicity induce the podocyte dysfunction, disrupting the integrity of the glomerular filtration barrier. This may be the initial step of diabetic nephropathy and the presence of albuminuria.42-44 The above-mentioned reasons might be possibly explained why the discriminative ability of BRI for the risk of UACR is superior to other adiposity indices.

In the clinical practice, it is convenient and highly cost-effective to use obesity-related indices to evaluate the visceral obesity and UACR risk. BRI showed the superior potential to be an accurate and stable indicator in the assessment of UACR. Hence, more attention should be paid to people with a high BRI to improve the distribution and deposition of visceral adiposity instead of only losing weight, in order to reduce CVD risk in clinical practice. We believe that taking $\mathrm{BRI}$ as part of management strategy is beneficial for reducing the diabetic nephropathy.

\section{Limitations}

Though the current study conducted in an aggregation of multi-community and the sample of our study was large, not only involved in 7 regions, but representing the general population in China. Several limitations, however, should be noted. Firstly, due to the design of this cross-sectional study, we can just investigate the association of different anthropometric indices with UACR and compared this relationship between new and traditional adiposity not cause. Second, though we excluded participants with related renal dysfunction and ACEI/ ARB medications, we did not detect other medications, possibly affecting the relationship. Furthermore, considering the convenience and price of measurements, we did not detect the distribution of body fat and abdominal fat percentage. Therefore, the conclusions should be draw with caution and more perfective studies should be needed to clarify the mechanisms underlying the relationship between anthropometric indices, especially $\mathrm{BRI}$, and albuminuria.

\section{Conclusions}

In conclusion, this current study found that newly developed visceral adiposity index BRI was more closely associated with UACR compared with traditional adiposity indices (BMI, WHR, WC) and showed a superior discriminative ability for UACR in the general Chinese population. Considering that BRI is an accurate indicator of visceral body fat and body shape, people with borderline blood glucose, normal blood pressure and eGFR should pay more attention to control body shape and visceral body fat to reduce the risk of albuminuria and slow CKD progression rather than just weight loss for a low BMI. It is significant to identify people at high risk of albuminuria to take effective measures to decrease the incidence of CKD in the early status.

\section{Declarations}

\section{Availability of data and materials}

The datasets used to support this study are not freely available due to participants' privacy protection. 


\section{Funding}

This work is supported by the National Key New Drug Creation and Manufacturing Program of Ministry of Science and Technology (2012ZX09303006-001), the National High Technology Research and Development Program of China (863 Program, 2011AA020107), Beijing Municipal Science Technology Commission (No. D141107005314004), National Natural Science Foundation of China (81300717) ,National Science and Technology Major Project 288 (2011ZX09307-001-08), the REACTION Study.

\section{Acknowledgments}

We would like to thank the participants and their families for participating in the study.

\section{Conflict of Interest}

The authors declare that the research was conducted in the absence of any commercial or financial relationships that could be construed as a potential conflict of interest.

\section{Authors' contributions:}

Jie Wang and Yun Wang have contributed equally to this work and share first authorship. Yiming Mu, Jie Wang and Yun Wang contributed to the conception and design of the study. Kang Chen, Binqi Li, Weiqing Wang, Zhengnan Gao, Xulei Tang, Li Yan, Qin Wan, Zuojie Luo, Guijun Qin and Lulu Chen recruited the subjects and supervised the study. Jie Wang , Yun Wang and Yang Liu analyzed the data and wrote the initial draft of the paper. Yiming Mu, Jie Wang and Yun Wang contributed to the writing, reviewing, and revising of the manuscript.

\section{References}

1. Levey AS, Becker C, Inker LA. Glomerular filtration rate and albuminuria for detection and staging of acute and chronic kidney disease in adults: a systematic review. Jama (2015) 313:837-46.

2. Romero-Aroca P, Baget-Bernaldiz M, Navarro-Gil R, et al. Glomerular Filtration Rate and/or Ratio of Urine Albumin to Creatinine as Markers for Diabetic Retinopathy: A Ten-Year Follow-Up Study. J Diabetes Res (2018) 2018:5637130.

3. Shogade TT, Essien IO, Ekrikpo UE, et al. Association of microalbuminuria with left ventricular dysfunction in Nigerian normotensive type 2 diabetes patients. Cardiovasc J Afr (2018) 29:283-8.

4. Zen M, Padmanabhan S, Cheung NW, et al. Microalbuminuria as an early predictor of preeclampsia in the pre-gestational diabetic population: A prospective cohort study. Pregnancy Hypertens (2019) 15:182-8.

5. Zhai Z, Feng J. Early microalbuminuria as a clinical marker for acute cerebral small vessel infarction. Neurol Res (2019) 41:151-5.

6. Zoccali C, Torino C, Tripepi G, Mallamaci F. Assessment of obesity in chronic kidney disease: what is the best measure? Curr Opin Nephrol Hypertens (2012) 21:641-6. 
7. Adams KF, Schatzkin A, Harris TB, et al. Overweight, obesity, and mortality in a large prospective cohort of persons 50 to 71 years old. N Engl J Med (2006) 355:763-78.

8. Xie L, Wang B, Jiang C, et al. BMI is associated with the development of chronic kidney diseases in hypertensive patients with normal renal function. J Hypertens (2018) 36:2085-91.

9. Fox CS, Larson MG, Leip EP, Culleton B, Wilson PW, Levy D. Predictors of new-onset kidney disease in a community-based population. Jama (2004) 291:844-50.

10. Garofalo C, Borrelli S, Minutolo R, Chiodini P, De Nicola L, Conte G. A systematic review and metaanalysis suggests obesity predicts onset of chronic kidney disease in the general population. Kidney Int (2017) 91:1224-35.

11. Yusuf $S$, Hawken S, Ounpuu S, et al. Obesity and the risk of myocardial infarction in 27,000 participants from 52 countries: a case-control study. Lancet (2005) 366:1640-9.

12. Britton KA, Massaro JM, Murabito JM, Kreger BE, Hoffmann U, Fox CS. Body fat distribution, incident cardiovascular disease, cancer, and all-cause mortality. J Am Coll Cardiol (2013) 62:921-5.

13. Fox CS, Massaro JM, Hoffmann U, et al. Abdominal visceral and subcutaneous adipose tissue compartments: association with metabolic risk factors in the Framingham Heart Study. Circulation (2007) 116:39-48.

14. Field AE, Coakley EH, Must A, et al. Impact of overweight on the risk of developing common chronic diseases during a 10-year period. Arch Intern Med (2001) 161:1581-6.

15. Janssen I, Katzmarzyk PT, Ross R. Body mass index is inversely related to mortality in older people after adjustment for waist circumference. J Am Geriatr Soc (2005) 53:2112-8.

16. Ross R, Berentzen T, Bradshaw AJ, et al. Does the relationship between waist circumference, morbidity and mortality depend on measurement protocol for waist circumference? Obes Rev (2008) 9:312-25.

17. Krakauer NY, Krakauer JC. Dynamic association of mortality hazard with body shape. PLoS One (2014) 9:e88793.

18. Ji M, Zhang S, An R. Effectiveness of A Body Shape Index (ABSI) in predicting chronic diseases and mortality: a systematic review and meta-analysis. Obes Rev (2018) 19:737-59.

19. Chang Y, Guo X, Chen Y, et al. A body shape index and body roundness index: two new body indices to identify diabetes mellitus among rural populations in northeast China. BMC Public Health (2015) 15:794.

20. Thomas DM, Bredlau C, Bosy-Westphal A, et al. Relationships between body roundness with body fat and visceral adipose tissue emerging from a new geometrical model. Obesity (Silver Spring) (2013) 21:2264-71.

21. Maessen MF, Eijsvogels TM, Verheggen RJ, Hopman MT, Verbeek AL, de Vegt F. Entering a new era of body indices: the feasibility of a body shape index and body roundness index to identify cardiovascular health status. PLoS One (2014) 9:e107212. 
22. Ning G. Risk Evaluation of cAncers in Chinese diabeTic Individuals: a IONgitudinal (REACTION) study. J Diabetes (2012) 4:172-3.

23. Liu B, Liu B, Wu G, Yin F. Relationship between body-roundness index and metabolic syndrome in type 2 diabetes. Diabetes Metab Syndr Obes (2019) 12:931-5.

24. Lin CC, $\mathrm{Li} \mathrm{Cl}$, Liu CS, et al. Risks of decreased renal function and increased albuminuria for glycemic status and metabolic syndrome components: Taichung Community Health study. Biomed Res Int (2014) 2014:841497.

25. Eckardt KU, Coresh J, Devuyst O, et al. Evolving importance of kidney disease: from subspecialty to global health burden. Lancet (2013) 382:158-69.

26. Bergman RN, Stefanovski D, Buchanan TA, et al. A better index of body adiposity. Obesity (Silver Spring) (2011)19:1083-9.

27. Gwynn RC, Berger M, Garg RK, Waddell EN, Philburn R, Thorpe LE. Measures of adiposity and cardiovascular disease risk factors, New York City Health and Nutrition Examination Survey, 2004. Prev Chronic Dis (2011) 8:A56.

28. Gómez-Ambrosi J, Silva C, Galofré JC, et al. Body mass index classification misses subjects with increased cardiometabolic risk factors related to elevated adiposity. Int J Obes (Lond) (2012) 36:28694.

29. Phillips CM, Tierney AC, Perez-Martinez P, et al. Obesity and body fat classification in the metabolic syndrome: impact on cardiometabolic risk metabotype. Obesity (Silver Spring) (2013) 21:E154-61.

30. Bibiloni Mdel M, Pons A, Tur JA. Defining body fatness in adolescents: a proposal of the AFAD-A classification. PLoS One (2013) 8:e55849.

31. Ashwell M, Gunn P, Gibson S. Waist-to-height ratio is a better screening tool than waist circumference and BMI for adult cardiometabolic risk factors: systematic review and meta-analysis. Obes Rev (2012) 13:275-86.

32. Savva SC, Tornaritis M, Savva ME, et al. Waist circumference and waist-to-height ratio are better predictors of cardiovascular disease risk factors in children than body mass index. Int $\mathrm{J}$ Obes Relat Metab Disord (2000) 24:1453-8.

33. Saqlain M, Akhtar Z, Karamat R, et al. Body Mass Index versus Other Adiposity Traits: Best Predictor of Cardiometabolic Risk. Iran J Public Health (2019) 48:2224-31.

34. Vazquez G, Duval S, Jacobs DR, Jr., Silventoinen K. Comparison of body mass index, waist circumference, and waist/hip ratio in predicting incident diabetes: a meta-analysis. Epidemiol Rev (2007) 29:115-28.

35. Janakiraman B, Abebe SM, Chala MB, Demissie SF. Epidemiology of General, Central Obesity and Associated Cardio-Metabolic Risks Among University Employees, Ethiopia: A Cross-Sectional Study. Diabetes Metab Syndr Obes (2020) 13:343-53.

36. Zhang N, Chang Y, Guo X, Chen Y, Ye N, Sun Y. A Body Shape Index and Body Roundness Index: Two new body indices for detecting association between obesity and hyperuricemia in rural area of China. Eur J Intern Med (2016) 29:32-6. 
37. Zhang K, Zhao Q, Li Y, et al. Feasibility of anthropometric indices to identify dyslipidemia among adults in Jilin Province: a cross-sectional study. Lipids Health Dis (2018) 17:16.

38. Chagnac A, Weinstein T, Korzets A, Ramadan E, Hirsch J, Gafter U. Glomerular hemodynamics in severe obesity. Am J Physiol Renal Physiol (2000) 278:F817-22.

39. Henegar JR, Bigler SA, Henegar LK, Tyagi SC, Hall JE. Functional and structural changes in the kidney in the early stages of obesity. J Am Soc Nephrol (2001) 12:1211-7.

40. Hall JE, Henegar JR, Dwyer TM, et al. Is obesity a major cause of chronic kidney disease? Adv Ren Replace Ther (2004) 11:41-54.

41. Sarafidis PA, Bakris GL. Non-esterified fatty acids and blood pressure elevation: a mechanism for hypertension in subjects with obesity/insulin resistance? J Hum Hypertens (2007) 21:12-9.

42. Kim JA, Montagnani M, Koh KK, Quon MJ. Reciprocal relationships between insulin resistance and endothelial dysfunction: molecular and pathophysiological mechanisms. Circulation (2006) 113:1888-904.

43. Bornfeldt KE, Tabas I. Insulin resistance, hyperglycemia, and atherosclerosis. Cell Metab (2011) 14:575-85.

44. Jauregui A, Mintz DH, Mundel P, Fornoni A. Role of altered insulin signaling pathways in the pathogenesis of podocyte malfunction and microalbuminuria. Curr Opin Nephrol Hypertens (2009) 18:539-45.

\section{Tables}

Table 1: Characteristics of the participants in different groups of UACR 


\begin{tabular}{|c|c|c|c|c|}
\hline Variable & Total & UACR $<30 \mathrm{mg} / \mathrm{g}$ & $\mathrm{UACR} \geq 30 \mathrm{mg} / \mathrm{g}$ & P-value \\
\hline $\mathrm{N}$ & 43591 & 37392 & 6199 & \\
\hline Age, years & $\begin{array}{l}57.51(52.19- \\
63.90)\end{array}$ & $\begin{array}{l}57.03(51.87- \\
63.01)\end{array}$ & $61.04(54.46-69.13)$ & $<0.001$ \\
\hline SBP, $\mathrm{mmHg}$ & $\begin{array}{l}131.00(118.00- \\
147.00)\end{array}$ & $\begin{array}{l}130.00(117.00- \\
145.00)\end{array}$ & $141.00(124.00-158.00)$ & $<0.001$ \\
\hline $\mathrm{DBP}, \mathrm{mmHg}$ & $\begin{array}{l}77.00(70.00- \\
85.00)\end{array}$ & $\begin{array}{l}77.00 \text { (70.00- } \\
85.00)\end{array}$ & $79.00(72.00-88.00)$ & $<0.001$ \\
\hline Pulse & $\begin{array}{l}79.00(72.00- \\
87.00)\end{array}$ & $\begin{array}{l}78.00(72.00- \\
86.00)\end{array}$ & $80.00(72.00-88.00)$ & $<0.001$ \\
\hline $\mathrm{FBG}, \mathrm{mmol} / \mathrm{L}$ & $5.53(5.11-6.18)$ & $5.50(5.10-6.10)$ & $5.80(5.21-6.90)$ & $<0.001$ \\
\hline PBG, mmol/L & $7.46(6.08-9.80)$ & $7.31(6.00-9.47)$ & $8.56(6.61-12.30)$ & $<0.001$ \\
\hline $\mathrm{HbA} 1 \mathrm{c}, \%$ & $5.90(5.60-6.30)$ & $5.90(5.60-6.20)$ & $6.10(5.70-6.70)$ & $<0.001$ \\
\hline$A L T, U / L$ & $\begin{array}{l}15.00(11.00- \\
21.00)\end{array}$ & $\begin{array}{l}15.00(11.00- \\
21.00)\end{array}$ & $15.00(11.00-22.00)$ & $<0.001$ \\
\hline AST, U/L & $\begin{array}{l}20.00(17.00- \\
24.00)\end{array}$ & $\begin{array}{l}20.00(17.00- \\
24.00)\end{array}$ & $21.00(17.00-26.00)$ & $<0.001$ \\
\hline GGT, U/L & $\begin{array}{l}20.00(15.00- \\
31.00)\end{array}$ & $\begin{array}{l}20.00(14.00- \\
31.00)\end{array}$ & $22.00(15.00-35.00)$ & $<0.001$ \\
\hline $\mathrm{TG}, \mathrm{mmol} / \mathrm{L}$ & $1.35(0.96-1.95)$ & $1.32(0.95-1.91)$ & $1.55(1.09-2.24)$ & $<0.001$ \\
\hline $\mathrm{TC}, \mathrm{mmol} / \mathrm{L}$ & $5.02(4.28-5.76)$ & $5.03(4.29-5.77)$ & $4.95(4.21-5.71)$ & $<0.001$ \\
\hline $\mathrm{LDL}, \mathrm{mmol} / \mathrm{L}$ & $2.91(2.33-3.53)$ & $2.92(2.34-3.54)$ & $2.82(2.23-3.43)$ & $<0.001$ \\
\hline $\mathrm{HDL}, \mathrm{mmol} / \mathrm{L}$ & $1.28(1.08-1.51)$ & $1.29(1.09-1.52)$ & $1.23(1.04-1.45)$ & $<0.001$ \\
\hline $\begin{array}{l}\text { eGFR, } \mathrm{ml} / \mathrm{min} \text { per } \\
1.73 \mathrm{~m}^{2}\end{array}$ & $\begin{array}{l}95.37 \text { (90.96- } \\
99.12)\end{array}$ & $\begin{array}{l}95.67(91.45- \\
99.41)\end{array}$ & $93.11(87.87-97.63)$ & $<0.001$ \\
\hline Sex, \% & & & & $<0.001$ \\
\hline Male & $13119(30.10 \%)$ & $11483(30.71 \%)$ & $1636(26.39 \%)$ & \\
\hline Female & $30472(69.90 \%)$ & 25909 (69.29\%) & $4563(73.61 \%)$ & \\
\hline Drinking status, $\%$ & & & & $<0.001$ \\
\hline No & 32666 (74.94\%) & 27706 (74.10\%) & $4960(80.01 \%)$ & \\
\hline $\begin{array}{l}\text { Occasional } \\
\text { drinkers }\end{array}$ & $8161(18.72 \%)$ & $7253(19.40 \%)$ & 908 (14.65\%) & \\
\hline
\end{tabular}




\begin{tabular}{|c|c|c|c|c|}
\hline Regular drinkers & $2764(6.34 \%)$ & $2433(6.51 \%)$ & \multicolumn{2}{|l|}{$331(5.34 \%)$} \\
\hline \multicolumn{4}{|l|}{ Smoking } & \multirow[t]{2}{*}{$<0.001$} \\
\hline No & $37222(85.39 \%)$ & $31804(85.06 \%)$ & $5418(87.40 \%)$ & \\
\hline $\begin{array}{l}\text { Occasional } \\
\text { smokers }\end{array}$ & $1357(3.11 \%)$ & $1198(3.20 \%)$ & \multicolumn{2}{|l|}{$159(2.56 \%)$} \\
\hline \multicolumn{2}{|c|}{ Regular smokers 5012 (11.50\%) } & $4390(11.74 \%)$ & \multicolumn{2}{|l|}{$622(10.03 \%)$} \\
\hline \multicolumn{2}{|c|}{ Cardiovascular 2371 (5.44\%) events } & $1824(4.88 \%)$ & $547(8.82 \%)$ & $<0.001$ \\
\hline Hypertension & $18293(41.97 \%)$ & $14571(38.97 \%)$ & $3722(60.04 \%)$ & $<0.001$ \\
\hline $\begin{array}{l}\text { Type } 2 \text { Diabetes } \\
\text { mellitus }\end{array}$ & 6642 (15.24\%) & $4903(13.11 \%)$ & $1739(28.05 \%)$ & $<0.001$ \\
\hline \multicolumn{5}{|c|}{ Anthropometric indices } \\
\hline $\mathrm{BMI}, \mathrm{kg} / \mathrm{m} 2$ & $\begin{array}{l}24.25(22.08- \\
26.57)\end{array}$ & $24.19(22.04-26.47)$ & $\begin{array}{l}24.71(22.46- \\
27.17)\end{array}$ & $<0.001$ \\
\hline BMI Z score & $\begin{array}{l}-0.06(-0.65- \\
0.57)\end{array}$ & $-0.08(-0.66-0.54)$ & $\begin{array}{l}0.06(-0.55- \\
0.73)\end{array}$ & $<0.001$ \\
\hline WC, $\mathrm{cm}$ & $\begin{array}{l}85.50(79.00- \\
93.00)\end{array}$ & $85.00(79.00-92.00)$ & $\begin{array}{l}88.00(80.50- \\
95.00)\end{array}$ & $<0.001$ \\
\hline WC Z score & $0.01(-0.64-0.75)$ & $-0.04(-0.64-0.65)$ & $\begin{array}{l}0.25(-0.49- \\
0.95)\end{array}$ & $<0.001$ \\
\hline WHR & $0.89(0.84-0.93)$ & $0.89(0.84-0.93)$ & $\begin{array}{l}0.89(0.85- \\
0.94)\end{array}$ & $<0.001$ \\
\hline WHR, Z score & $0.01(-0.61-0.61)$ & $-0.01(-0.62-0.59)$ & $\begin{array}{l}0.12(-0.49- \\
0.73)\end{array}$ & $<0.001$ \\
\hline WHtR & $0.53(0.49-0.58)$ & $0.53(0.49-0.57)$ & $\begin{array}{l}0.55(0.51- \\
0.59)\end{array}$ & $<0.001$ \\
\hline WHtR Z score & $\begin{array}{l}-0.01(-0.65- \\
0.66)\end{array}$ & $-0.05(-0.67-0.60)$ & $\begin{array}{l}0.23(-0.45- \\
0.93)\end{array}$ & $<0.001$ \\
\hline BRI & $3.93(3.16-4.82)$ & $3.88(3.14-4.75)$ & $\begin{array}{l}4.25(3.39- \\
5.21)\end{array}$ & $<0.001$ \\
\hline BRI Z score & $\begin{array}{l}-0.07(-0.66- \\
0.60)\end{array}$ & $-0.11(-0.67-0.54)$ & $\begin{array}{l}0.17(-0.48- \\
0.89)\end{array}$ & $<0.001$ \\
\hline
\end{tabular}

Data of characteristics expressed as median (25th percentile-75th percentile) for continuous variables of non-normal distribution and percentage (\%) for categorical variables. SBP: systolic blood pressure; DBP: diastolic blood pressure; eGFR: estimated glomerular filtration rate; TG: triglyceride; TC: total cholesterol; LDL: low-density lipoprotein cholesterol; HDL: high-density lipoprotein cholesterol; ALT: alanine aminotransferase; AST: aspartate aminotransferase GGT: gamma-glutamyl transpeptidase; FBG: 0-hour 
fasting blood glucose; PBG: 2-hour postprandial blood glucose; HbA1c: haemoglobin A1c; BMI: body mass index; WC: waist circumstance; WHR: waist-to-hip ratio; WHtR: waist-to-height ratio; ABSI: a body shape index; BRI: body roundness index.

Table 2: Associations between obesity indicators and UACR in total population

\begin{tabular}{lll} 
Variable $\quad$ Non-adjusted & Model I & Model II \\
\hline OR $(95 \% \mathrm{Cl})$ P-value & OR $(95 \% \mathrm{Cl})$ P-value & OR $(95 \% \mathrm{Cl})$ P-value
\end{tabular}

\begin{tabular}{|c|c|c|c|}
\hline \multicolumn{2}{|c|}{ traditional indices of adiposity } & \multirow[t]{2}{*}{$1.12(1.09,1.15)<0.0001$} & \multirow[t]{2}{*}{$1.00(0.97,1.04) 0.9093$} \\
\hline BMI Z score & $1.14(1.11,1.17)<0.0001$ & & \\
\hline WHR Z score & $1.15(1.12,1.18)<0.0001$ & $1.07(1.04,1.10)<0.0001$ & $1.00(0.96,1.04) 0.9689$ \\
\hline WC Z score & $1.24(1.20,1.27)<0.0001$ & $1.17(1.14,1.20)<0.0001$ & $1.03(0.98,1.08) 0.2827$ \\
\hline \multicolumn{2}{|c|}{ newly developed indices of adiposity } & $1.16(1.13,1.19)<0.0001$ & $1.08(1.02,1.13) 0.0075$ \\
\hline WHtR Z score & $1.30(1.27,1.34)<0.0001$ & & \\
\hline BRI Z score & $1.29(1.25,1.32)<0.0001$ & $1.15(1.12,1.18)<0.0001$ & $1.22(1.11,1.33)<0.0001$ \\
\hline
\end{tabular}

Non-adjusted model for none.

Model I adjusted for age and sex.

Model II adjusted for age, sex, BMI, center, SBP, DBP, pulse, LDL, HDL, TG, FBG, PBG, HbA1c, ALT, AST, GGT, eGFR, BMI Z score, WHR Z score, ABSI Z score, BRI Z score, smoking status, drinking status, history of hypertension, cardiovascular history, diabetes history, medication history.

Table 3: Associations between anthropometric indices and UACR in different subgroups 
Subgroups

\begin{tabular}{lllll} 
BMI Z score & $\begin{array}{l}\text { WHR Z } \\
\text { score }\end{array}$ & WC Z score & $\begin{array}{l}\text { WHtR Z } \\
\text { score }\end{array}$ & BRI Z score \\
\hline OR $(95 \% \mathrm{Cl})$ & $\begin{array}{l}\text { OR }(95 \mathrm{Cl}) \mathrm{P}- \\
\text { value }\end{array}$ & $\begin{array}{l}\text { OR }(95 \% \mathrm{Cl}) \\
\text { P-value }\end{array}$ & $\begin{array}{l}\text { OR }(95 \% \mathrm{Cl}) \\
\text { P-value }\end{array}$ & $\begin{array}{l}\text { OR }(95 \% \mathrm{Cl}) \\
\text { P-value }\end{array}$
\end{tabular}

Age

\begin{tabular}{llllll} 
Age $<55$ years & 1.01 & 1.04 & 0.99 & 1.08 & 1.41 \\
& $(0.95,1.08)$ & $(0.97,1.12)$ & $(0.90,1.10)$ & $(0.98,1.20)$ & $(1.20,1.66)$ \\
& 0.7443 & 0.2809 & 0.9051 & 0.1370 & $<0.0001$ \\
\hline $55 \geq$ age<64years & 0.99 & 0.97 & 1.01 & 1.05 & 1.19 \\
& $(0.93,1.05)$ & $(0.90,1.04)$ & $(0.93,1.10)$ & $(0.96,1.14)$ & $(1.03,1.38)$ \\
& 0.7063 & 0.3372 & 0.7461 & 0.2800 & 0.0203 \\
\hline Age $\geq$ 65 years & 1.00 & 1.00 & 1.08 & 1.10 & 1.11 \\
& $(0.94,1.07)$ & $(0.93$, & $(0.98,1.18)$ & $(1.00,1.20)$ & $(0.95,1.29)$ \\
& 0.8948 & $1.08) 0.9444$ & 0.1198 & 0.0491 & 0.2096
\end{tabular}

Sex

\begin{tabular}{llllll} 
men & 1.01 & 1.01 & 1.09 & 1.13 & 1.16 \\
& $(0.93,1.09)$ & $(0.92,1.10)$ & $(0.98,1.22)$ & $(1.00,1.28)$ & $(0.95,1.40)$ \\
\multirow{2}{*}{ women } & 0.8322 & 0.8486 & 0.1160 & 0.0495 & 0.1411 \\
& 1.01 & 1.01 & 1.03 & 1.08 & 1.29 \\
& $(0.97,1.05)$ & $(0.96,1.05)$ & $(0.97,1.09)$ & $(1.02,1.15)$ & $(1.16,1.43)$ \\
& 0.7829 & 0.7883 & 0.3963 & 0.0083 & $<0.0001$
\end{tabular}

Hypertension

$\begin{array}{llllll}\text { Yes } & 1.07 & 1.00 & 1.05 & 1.09 & 1.14 \\ & (1.02,1.12) & (0.95,1.06) & (0.98,1.13) & (1.01, & (1.01,1.28) \\ \text { No } & 0.0075 & 0.9490 & 0.1518 & 1.17) 0.0242 & 0.0341 \\ & 0.95 & 1.01 & 1.01 & 1.07 & 1.29 \\ & (0.89,1.00) & (0.95, & (0.94,1.10) & (0.99,1.16) & (1.12,1.48) \\ & 0.0645 & 1.07) 0.7085 & 0.7341 & 0.0790 & 0.0004\end{array}$

Diabetes

$\begin{array}{llllll}\text { Yes } & 1.05 & 1.06 & 1.11 & 1.20 & 1.33 \\ & (0.99,1.12) & (0.98,1.14) & (1.01,1.23) & (1.09,1.33) & (1.13,1.57) \\ & 0.1061 & 0.1524 & 0.0313 & 0.0004 & 0.0007\end{array}$

No

$\begin{array}{lllll}1.03 & 1.01 & 1.05 & 1.09 & 1.17\end{array}$



$(1.00,1.07)$
0.0823
$(0.97,1.06)$
0.5021
$(1.00,1.11)$
0.0508
$(1.03,1.15)$
0.0023
$(1.07,1.28)$
0.0009

Model Il adjusted for age, sex, BMI, center, SBP, DBP, pulse, LDL, HDL, TG, FBG, PBG, HbA1c, ALT, AST, GGT, eGFR, BMI Z score, WHR Z score, ABSI Z score, BRI Z score, smoking status, drinking status, history of hypertension, cardiovascular history, diabetes history, medication history.

\section{Table 4: Associations between anthropometric indices and UACR in IFG, IGT, IFG+IGT}

\begin{tabular}{|c|c|c|c|}
\hline \multirow[t]{2}{*}{ Subgroups } & IFG & IGT & $\mathrm{IFG}+\mathrm{IGT}$ \\
\hline & OR (95\% Cl) P-value & OR (95\% Cl) P-value & OR (95\% Cl) P-value \\
\hline \multicolumn{2}{|c|}{ traditional anthropometric indices } & $0.99(0.95,1.03) 0.6455$ & $1.10(1.00,1.21) 0.0595$ \\
\hline BMI Z score & $1.04(0.89,1.20) 0.6419$ & & \\
\hline WHR Z score & $1.00(0.82,1.22) 0.9873$ & $1.00(0.96,1.05) 0.8413$ & $0.97(0.87,1.08) 0.5799$ \\
\hline WC Z score & $1.24(0.97,1.58) 0.0801$ & $1.02(0.96,1.08) 0.5168$ & $1.02(0.89,1.17) 0.8046$ \\
\hline \multicolumn{2}{|c|}{ newly developed anthropometric indices } & $1.06(1.00,1.12) 0.0669$ & $1.08(0.94,1.24) 0.2676$ \\
\hline
\end{tabular}

WHtR Z score $1.19(0.91,1.55) 0.2116$

BRI Z score $\quad 0.84(0.53,1.34) 0.4717 \quad 1.20(1.08,1.33) 0.0005 \quad 1.34(1.03,1.74) 0.0320$

Model Il adjusted for age, sex, BMI, center, SBP, DBP, pulse, LDL, HDL, TG, FBG, PBG, HbA1c, ALT, AST, GGT, eGFR, BMI Z score,WHR Z score, ABSI Z score, BRI Z score, smoking status,drinking status, history of hypertension, cardiovascular history, diabetes history, medication history.

Table 5: Associations between anthropometric indices and UACR in different subgroups of eGFR

\begin{tabular}{|c|c|c|}
\hline \multirow[t]{2}{*}{ Subgroups } & eGFR $<90$ & $\mathrm{eGFR} \geq 90$ \\
\hline & OR $(95 \% \mathrm{Cl}) \mathrm{P}$-value & OR (95\% Cl) P-value \\
\hline \multicolumn{2}{|c|}{ traditional anthropometric indices } & $1.01(0.97,1.06) 0.5514$ \\
\hline BMI Z score & $0.98(0.91,1.05) 0.5201$ & \\
\hline WHR Z score & $1.01(0.94,1.09) 0.7590$ & $0.99(0.95,1.04) 0.7812$ \\
\hline WC Z score & $1.09(0.99,1.20) 0.0768$ & $1.01(0.95,1.07) 0.8554$ \\
\hline \multicolumn{2}{|c|}{ newly developed anthropometric indices } & $1.06(0.99,1.13) 0.0980$ \\
\hline WHtR Z score & $1.12(1.01,1.23) 0.0235$ & \\
\hline BRI Z score & $1.12(0.95,1.31) 0.1708$ & $1.26(1.13,1.41)<0.0001$ \\
\hline
\end{tabular}


Model II adjusted for age, sex, BMI, center, SBP, DBP, pulse, LDL, HDL, TG, FBG, PBG, HbA1c, ALT, AST, GGT, eGFR, BMI Z score, WHR Z score, ABSI Z score, BRI Z score, smoking status, drinking status, history of hypertension, cardiovascular history, diabetes history, medication history.

\section{Figures}

Participants from 7 centers of Reaction study: Dalian 10140, Lanzhou 10026, Guangzhou 9743, Luzhou 8105,

Shanghai 6821, Zhengzhou 1978, Wuhan 995

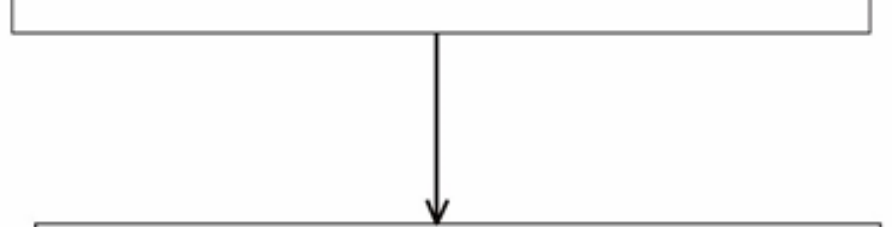

A total of 47808 participants aged 40 years or order were recruited

Excluded participants:

1. Previous history of drugs used ACEI drugs $(\mathbf{n}=595)$

ARB drugs $(\mathrm{n}=1099)$

2: Kidncy related discases Kidney stones $(n=1541)$ nephrotic syndrome $(\mathrm{n}=17)$ chronic diseases $(\mathrm{n}=227)$ other kidney diseases $(\mathbf{n}=228)$

3. participants without other complete data $(n=1243)$ 
Flow chart of the selection of study participants

Page 21/21 